\title{
Linear magnetoresistance in mosaic-like bilayer graphene
}

\author{
Ferdinand Kisslinger ${ }^{1}$, Christian Ott ${ }^{1}$, Christian Heide ${ }^{1}$, Erik Kampert ${ }^{2}$, Benjamin Butz ${ }^{3}$, \\ Erdmann Spiecker ${ }^{3}$, Sam Shallcross ${ }^{4}$ and Heiko B. Weber ${ }^{1 \star}$
}

\begin{abstract}
The magnetoresistance of conductors usually has a quadratic dependence on magnetic field', however, examples exist of non-saturating linear behaviour in diverse materials $s^{2-6}$. Assigning a specific microscopic mechanism to this unusual phenomenon is obscured by the co-occurrence and interplay of doping, mobility fluctuations and a polycrystalline structure ${ }^{7,8}$. Bilayer graphene has virtually no doping fluctuations, yet provides a built-in mosaic tiling due to the dense network of partial dislocations ${ }^{9,10}$. We present magnetotransport measurements of epitaxial bilayer graphene that exhibits a strong and reproducible linear magnetoresistance that persists to $B=62 \mathrm{~T}$ at and above room temperature, decorated by quantum interference effects at low temperatures. Partial dislocations thus have a profound impact on the transport properties in bilayer graphene, a system that is frequently assumed to be dislocation-free. It further provides a clear and tractable model system for studying the unusual properties of mosaic conductors.
\end{abstract}

Although most real materials exhibit a quadratic magnetoresistance (MR), linear MR has been observed, even at room temperature, in conductors as varied as three-dimensional (3D) silver chalcogenides, semiconductors, topological insulators, and 2D multilayer graphenes ${ }^{2-6,11-14}$. Several theories have been developed seeking a general explanation of this phenomenon. A classical mechanism is suspected that mixes the transverse Hall resistance, which is linear in magnetic field, into the longitudinal resistance ${ }^{1}$. In particular, inhomogeneities may allow local Hall currents that in turn modify the potential landscape. The most rigorous model to treat this local Hall current map is that introduced by Parish and Littlewood (PL) in 2003, which represents the material as a mosaic of four-terminal interconnected conductive discs. This model has been invoked to explain linear MR in a number of experimental contexts. Nevertheless, an experiment which links the essence of this insightful but simple model to a real material is still lacking.

To make a conceptually clear experiment in the spirit of $\mathrm{PL}$ it is first advantageous to treat a $2 \mathrm{D}$ system. Not only is magnetotransport an essentially $2 \mathrm{D}$ phenomenon, but also the mosaic nature may then in principle be entirely imaged. An essential requirement for a clear experimental realization of the PL model is a material for which the mosaic grains possess negligible internal structure-that is, a constant charge density, mobility and weak magnetoresistance. Furthermore, the granular structure and interconnect topology should be fully characterized. For such an experiment, bilayer epitaxial graphene is an ideal choice as it combines two-dimensionality with fixed charge density (due to the epitaxially defined substrate ${ }^{15}$ ) and a room-temperature magnetoresistance expected to be weak $^{16}$. Recently, we have discovered that epitaxial bilayer graphene, despite being a conductor with a good overall mobility, is threaded by a dense network of wellcharacterized partial dislocations ${ }^{10}$. It turns out that it is the perfect realization of a granular material for a test of the PL model. Its simplicity can be contrasted with previous experimental studies in which linear MR was observed and a connection to the PL model was made. This includes studies on polycrystalline semiconductors ${ }^{17}$ and silver chalcogenides ${ }^{3,4}$, where the influence of significant charge density fluctuations, mobility fluctuations, and the all-important granular structure can barely be separated. Nevertheless, the stateof-the-art understanding makes a specific choice and links linear MR to mobility fluctuations ${ }^{6,12,13,17}$ (a point focused on in the original PL papers), neglecting the crucial role played by material granularity. Indeed, in our material the very concept of a distribution of individual segment mobilities is not physically defined, as the mean free path of $\sim 50 \mathrm{~nm}$ is comparable to the grain size (see Supplementary Information). The present experiment, therefore, takes the opposite direction, in that the grain shape is the only remaining degree of freedom that provides inhomogeneities.

Linear MR has been observed in multilayer graphene, where it was (incorrectly) assigned to a quantum magnetoresistance effect ${ }^{18}$ in the extreme quantum limit ${ }^{19}$, and in exfoliated bilayer graphene flakes in a limited field region ${ }^{20}$. The present study on epitaxial bilayer graphene goes far beyond these experiments. It shows a strong and fully reproducible linear MR up to the highest fields, and a convincing assignment to a mosaic-like conductor.

For the investigation of intrinsic charge transport in graphene, it has been a good strategy to investigate large-area samples. In this way, the strong influence of edge effects and universal conductance fluctuations (UCF) that often dominate the MR in small flakes may be avoided. As a consequence, a nearly complete quantitative understanding of both the temperature-dependent parabolic MR below the quantum limit, as caused by electronelectron interaction (EEI) and weak localization (WL), has been obtained in monolayer graphene ${ }^{16}$.

In this paper, we consider bilayer graphene, more specifically hydrogen-intercalated epitaxial bilayer graphene on $\mathrm{SiC}(0001)$ (refs 21,22) (eBLG) with a charge carrier density of

\footnotetext{
${ }^{1}$ Lehrstuhl für Angewandte Physik, Friedrich-Alexander-Universität Erlangen-Nürnberg (FAU), Staudtstraße 7, 91058 Erlangen, Germany. ${ }^{2}$ Dresden High Magnetic Field Laboratory, Helmholtz-Zentrum Dresden-Rossendorf, Bautzner Landstraße 400, 01328 Dresden, Germany. ${ }^{3}$ Lehrstuhl für Mikro- und Nanostrukturforschung \& Center for Nanoanalysis and Electron Microscopy (CENEM), Friedrich-Alexander-Universität Erlangen-Nürnberg (FAU), Cauerstraße 6, 91058 Erlangen, Germany. ${ }^{4}$ Lehrstuhl für Theoretische Festkörperphysik, Friedrich-Alexander-Universität Erlangen-Nürnberg (FAU), Staudtstraße 7, 91058 Erlangen, Germany. *e-mail: heiko.weber@fau.de
} 
a

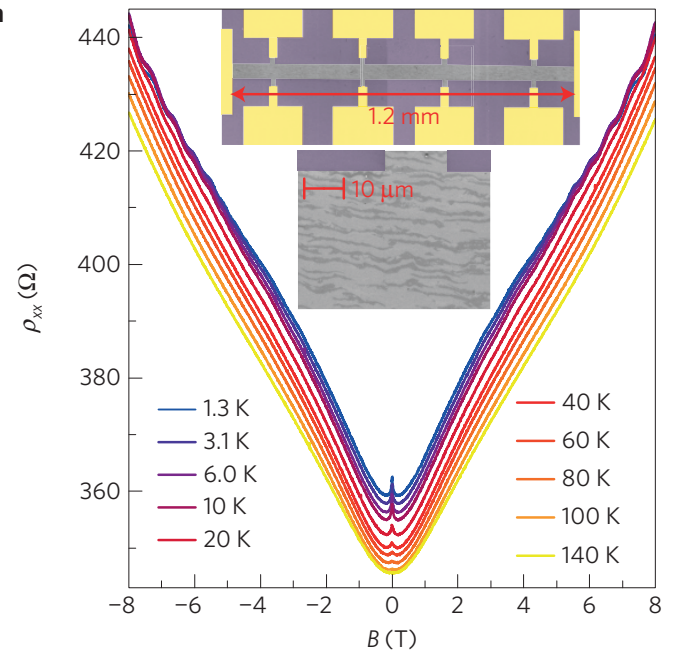

b

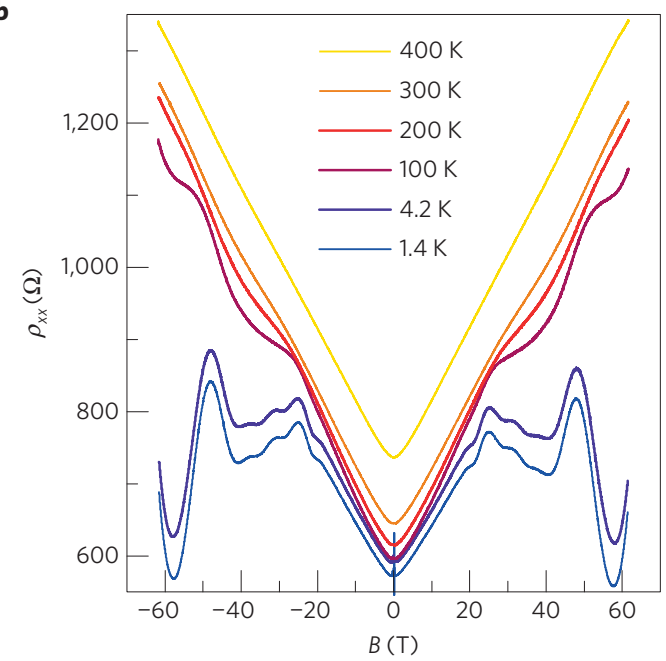

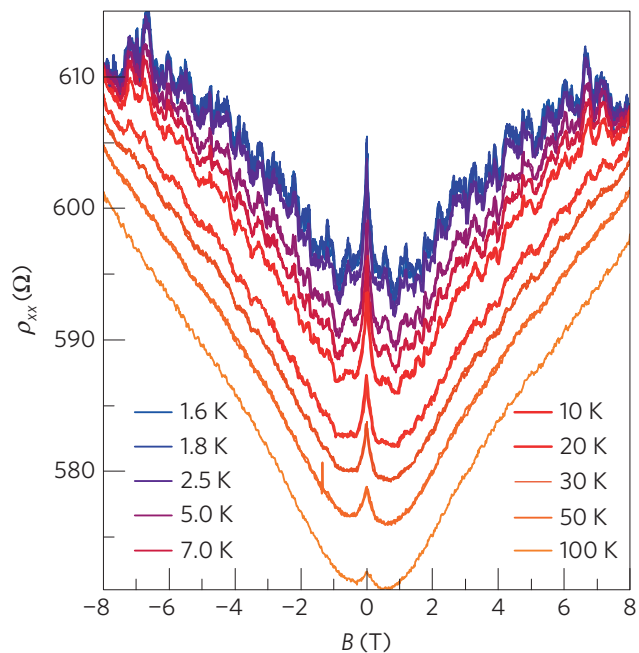

d

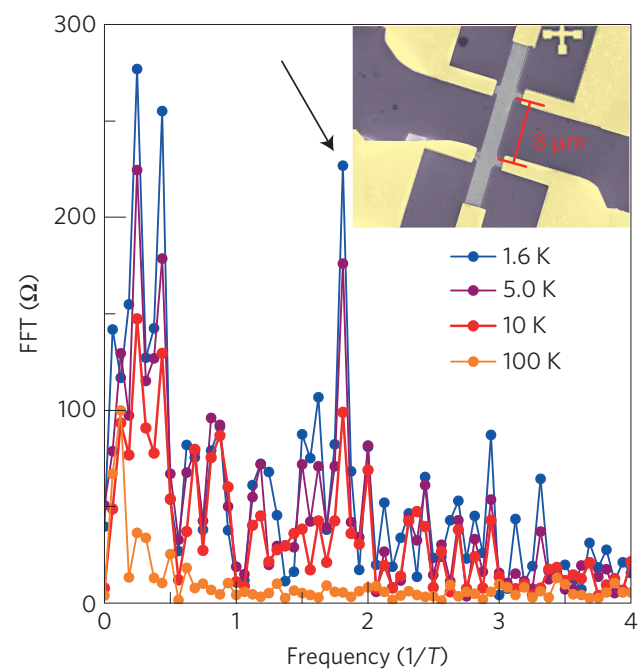

Figure 1 | Experimental data of linear MR. a, In large-area bilayer graphene (the scanning electron micrograph (SEM) of a Hall bar is shown in the inset, together with a close-up that shows mainly bilayer, but also trilayer strips as darker shaded areas), a strong linear MR contribution appears in addition to the temperature-dependent WL and EEl corrections similarly observed in monolayer graphene. $\mathbf{b}, \mathrm{MR}$ measured in pulsed magnetic fields, showing non-saturating linear behaviour up to $62 \mathrm{~T}$ at room temperature, with quantum deviations at lower temperatures (data have been vertically shifted by $20 \Omega$ for better visibility). c,d, A smaller, pure bilayer sample exhibits linear MR and further periodic quantum interference fluctuations at low temperatures (c), the Fourier spectrum of which shows a correlation field of $B_{c}=0.55 \mathrm{~T}$, indicated by the arrow (d). Inset in $\mathbf{d}$ is a SEM image of the sample showing the absence of trilayer areas.

$p \approx 7 \times 10^{12} \mathrm{~cm}^{-2}$. On first sight, one would expect very similar behaviour in this material ${ }^{23}$. Surprisingly, the MR is qualitatively different: Fig. 1a shows data of the MR $\rho(B, T)$ for eBLG. Apart from the WL correction in small fields $B \leq 0.5 \mathrm{~T}$, the expected parabolic $\Delta \rho_{\mathrm{EEI}}(B)$ appears, the curvature of which is temperature dependent ${ }^{16}$. In addition, a linear $\Delta \rho_{\text {lin }}(B)$ occurs, which is the main topic of this manuscript. Figure $1 \mathrm{~b}$ shows measurements of $\rho(B)$ in a pulsed magnetic field experiment, with fields up to $B=62 \mathrm{~T}$. The most striking findings are: the linear MR does not saturate up to the highest fields and the linear MR is essentially temperature independent, which suggests a classical mechanism. Consequently, we focus on the conceptual framework of PL (ref. 7), based on a mosaic-like sample. For identification of the tiling, a closer look at the structure is mandatory.

When comparing monolayer with bilayer graphene, there is one structural property present in bilayer graphene the importance of which has been discussed only very recently: the twofold degeneracy with respect to stacking. Bilayer graphene is Bernal stacked, which leaves two possibilities, typically referred to as $\mathrm{AB}$ and $\mathrm{AC}$ stacking. Although the two resulting structures are equivalent and result in the same electronic properties ${ }^{23}$, the interface lines between the two, termed 'partial dislocations' ${ }^{\text {'0 }}$ or 'strain solitons', act as scattering lines, which will be shown below. Partial dislocations are ubiquitous in bilayers and have remarkable impact at the charge neutrality point, resulting in overall insulating behaviour ${ }^{24}$. In the given material, eBLG, dense dislocation networks have been observed recently by dark-field transmission electron microscopy (TEM; ref. 10) after removing the $\mathrm{SiC}$ substrate ${ }^{25}$. Figure 2 a shows a superposition of three distinct dark-field TEM images exhibiting a real partial dislocation network, taken from ref. 10, in which Bernalstacked areas range from almost regular to highly distorted triangles.

To investigate the scattering properties of these partial dislocations we have performed calculations using an effective Dirac-Weyl approach in which the dislocation network enters as an off-diagonal effective field $\mathbf{S}(\mathbf{r})$ in a $4 \times 4$ Hamiltonian, in which the diagonal $2 \times 2$ blocks contain the Dirac-Weyl operators of each layer ${ }^{23}$. We choose $\mathbf{S}(\mathbf{r})$ to represent the partial dislocation array shown in Fig. 2b, which represents a micrometre-sized cutout of the TEM image of Fig. 2a. The line colour identifies the three distinct partial dislocations with their corresponding Burgers 
a

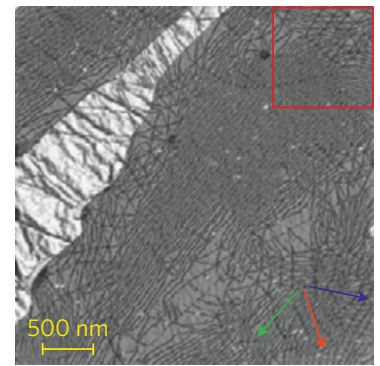

C


e


Figure 2 | From network structure to linear MR. a, Partial dislocation network in a bilayer membrane originating from epitaxial graphene on $\mathrm{SiC}(0001)$. The image shows a composite (or superposition) of three dark-field TEM images, taken with the three $\{11 \overline{2} 0\}$ reflections, such that the full network of partial dislocations is revealed, providing the mosaic tiling of bilayer graphene. $\mathbf{b}$, Partial dislocation network in which the colour encodes the crystallographic Burgers vector associated with the partial dislocations as extracted from the individual dark-field TEM data (red square in $\mathbf{a})^{10}$. c, Probability $|\Psi|^{2}$ of a network eigenstate, which for clarity we select close to the Dirac point (the energy of the eigenstate is indicated by the dashed vertical line in $\mathbf{d}$ ); segmentation of the network state on the $A B / A C$ tiles is evident. $\mathbf{d}$, Spectral weight of the monolayer graphene states present in the network state; for a perfect $A B$ bilayer only two such states would be present. e, This motivates the classical model of a network of three-terminal interconnected conductive discs, for which at finite magnetic field the resulting potential landscape (colour map) and non-trivial current distribution (green arrows) resembles that found for the four-terminal case $7 . \mathbf{f}$, Comparison of MR for a $(4 \times 3)$ network (see e) with a similar but larger $(32 \times 24)$ network, for which we assumed a charge carrier density of $6 \times 10^{12} \mathrm{~cm}^{-2}$ and a mobility of $10,000 \mathrm{~cm}^{2} \mathrm{~V}^{-1} \mathrm{~s}^{-1}$. The first value is measured, whereas the second is a reasonable assumption that gives a crossover field to linear MR similar to experiments.

vectors shown in Fig. 2a. Solving the quantum problem numerically for this specific geometry reveals electron wavefunctions localized on individual tiles of the mosaic-like network, as seen in Fig. 2c. This is associated with multiple scattering through a network of semi-transparent partial dislocations, a point of view supported by the spectral decomposition of the network wavefunction into single-layer graphene states (Fig. 2d). This is the driving mechanism that divides the bilayer sheet into a network of weakly connected segments, precisely the physics that underpins the PL model.

To check whether linear MR is present in this geometry, we implemented a numerical simulation conceptually similar to the PL model, now on a triangular network with threefold interconnected segments. The linear MR calculated for this network is essentially that of the fourfold PL network. To model the network disorder observed in Fig. 2a,b, we introduce topological defects by removing interconnects or introducing shortcuts in the network (see Supplementary Information). We thus have convincing evidence from the simulations that the linear MR is a robust finding in mosaic materials and that this feature results from the segmentation of the network wavefunctions.

An additional source of disorder present in all large-area eBLG samples is the existence of trilayer areas (about $20 \%$ of the sample), which introduce a further stacking degree of freedom ( $A B C$ versus ABA stacking). As a blind experiment, we prepared a sample containing only bilayer graphene. This sample is smaller $\left(2 \times 8 \mu \mathrm{m}^{2}\right)$ and, therefore, provides finite-size mesoscopic effects, which are avoided in the previous samples. Figure 1c shows the MR data obtained with this sample. The finding of linear MR persists at room temperature, which proves unambiguously that indeed the bilayer (not the trilayer) is the relevant source of linear MR. On cooling the sample to low temperatures the resistance exhibits reproducible fluctuations, absent in the large-area samples. These fluctuations contain both the expected aperiodic UCF contribution as well as an unexpected and significant periodic component, with a periodicity of $B_{c}=0.55 \mathrm{~T}$ (indicated by the arrow in Fig. 1d). This is certainly a quantum phenomenon, as it occurs only at low temperatures $(T<20 \mathrm{~K})$. In the framework of single-particle quantum interference, $B_{c}$ can be linked to a flux-threaded loop of area $A_{\mathrm{c}}=\Phi_{0} / B_{\mathrm{c}}$ (with $\Phi_{0}=h / e$ being the flux quantum). The numerical value of this area is $\sim(90 \mathrm{~nm})^{2}$. Although the irregular nature of the partial network precludes a clear assignment to a specific geometry, $(90 \mathrm{~nm})^{2}$ corresponds well with the average area of the regular triangles in Fig. 2b. The underpinning of both TEM and the conductance oscillations by the same length scale strongly suggests that charge carrier motion is sensitive to features of the partial dislocation network. When discussing the large-area samples it is remarkable that the linear $\mathrm{MR}$ in the $1-20 \mathrm{~T}$ range persists at $1.4 \mathrm{~K}$, a regime in which strong quantum Hall signatures are usually expected to be observed. Note that linear longitudinal MR may occur in the quantum Hall regime, when resistivity fluctuations are present $^{26}$. In our experiment, we suspect that this classical behaviour is due to the insensitivity of the mosaic network to the spectra of the individual tiles. Indeed, replacing the classical treatment of the discs by a multi-terminal edge-state model yields again a linear MR in our simulations, which will be explored in further investigations.

To conclude, we have identified bilayer graphene as the simplest mosaic-like conductor so far, in which segmentation of the electronic structure is the only ingredient that leads to classical linear magnetoresistance. This phenomenon can now convincingly be traced back to rather simple models. This will pave the way to understand charge transport in emergent $2 \mathrm{D}$ materials and provides a prototypical system for further study of the impact of material segmentation on electronic structure.

This experiment also sheds new light on recent experiments that were interpreted assuming virtually perfect bilayer graphene samples, and disregarding partial dislocations ${ }^{27-30}$. It is at least conceivable that some of the spectral features observed can be traced back to the physics of partial dislocations near charge neutrality. We expect a rich variety of behaviour between dislocation-free samples and the robust finding of a linear MR for a large-area partial dislocation network.

\section{Methods}

Methods and any associated references are available in the online version of the paper.

Received 4 November 2014; accepted 15 May 2015; published online 22 June 2015 


\section{References}

1. Pippard, A. B. Magnetoresistance in Metals (Cambridge Univ. Press, 1989).

2. Xu, R. et al. Large magnetoresistance in non-magnetic silver chalcogenides. Nature 390, 57-60 (1997).

3. Lee, M., Rosenbaum, T. F., Saboungi, M. L. \& Schnyders, H. S. Band-gap tuning and linear magnetoresistance in the silver chalcogenides. Phys. Rev. Lett. 88, 066602 (2002).

4. Husmann, A. et al. Megagauss sensors. Nature 417, 421-424 (2002).

5. Delmo, M. P., Yamamoto, S., Kasai, S., Ono, T. \& Kobayashi, K. Large positive magnetoresistive effect in silicon induced by the space-charge effect. Nature 457, 1112-1115 (2009).

6. Kozlova, N. V. et al. Linear magnetoresistance due to multiple-electron scattering by low-mobility islands in an inhomogeneous conductor. Nature Commun. 3, 1097 (2012).

7. Parish, M. M. \& Littlewood, P. B. Non-saturating magnetoresistance in heavily disordered semiconductors. Nature $\mathbf{4 2 6}$ 162-165 (2003)

8. Parish, M. M. \& Littlewood, P. B. Classical magnetotransport of inhomogeneous conductors. Phys. Rev. B 72, 094417 (2005).

9. Alden, J. S. et al. Strain solitons and topological defects in bilayer graphene. Proc. Natl Acad. Sci. USA 110, 11256-11260 (2013).

10. Butz, B. et al. Dislocations in bilayer graphene. Nature 505, 533-537 (2014).

11. Porter, N. A. \& Marrows, C. H. Linear magnetoresistance in n-type silicon due to doping density fluctuations. Sci. Rep. 2, 565 (2012).

12. Wang, W. et al. Large linear magnetoresistance and Shubnikov-de Hass oscillations in single crystals of YPdBi Heusler topological insulators. Sci. Rep. 3, 2181 (2013).

13. Assaf, B. A. et al. Linear magnetoresistance in topological insulator thin films: Quantum phase coherence effects at high temperatures. Appl. Phys. Lett. 102, 012102 (2013).

14. Wang, Z. H. et al. Granularity controlled nonsaturating linear magnetoresistance in topological insulator $\mathrm{Bi}_{2} \mathrm{Te}_{3}$ films. Nano Lett. 14, 6510-6514 (2014).

15. Kim, K. S. et al. Coexisting massive and massless Dirac fermions in symmetry-broken bilayer graphene. Nature Mater. 12, 887-892 (2013).

16. Jobst, J., Waldmann, D., Gornyi, I. V., Mirlin, A. D. \& Weber, H. B. Electron-electron interaction in the magnetoresistance of graphene. Phys. Rev. Lett. 108, 106601 (2012).

17. Hu, J. \& Rosenbaum, T. F. Classical and quantum routes to linear magnetoresistance. Nature Mater. 7, 697-700 (2008).

18. Friedman, A. L. et al. Quantum linear magnetoresistance in multilayer epitaxial graphene. Nano Lett. 10, 3962-3965 (2010).

19. Abrikosov, A. A. Quantum magnetoresistance. Phys. Rev. B 58, 2788-2794 (1998).

20. Zhi-Min, L., Yang-Bo, Z., Han-Chun, W., Bing-Hong, H. \& Da-Peng, Y. Observation of both classical and quantum magnetoresistance in bilayer graphene. Europhys. Lett. 94, 57004 (2011).
21. Riedl, C., Coletti, C., Iwasaki, T., Zakharov, A. A. \& Starke, U. Quasi-free-standing epitaxial graphene on $\mathrm{SiC}$ obtained by hydrogen intercalation. Phys. Rev. Lett. 103, 246804 (2009).

22. Speck, F. et al. The quasi-free-standing nature of graphene on $\mathrm{H}$-saturated SiC(0001). Appl. Phys. Lett. 99, 122106 (2011).

23. McCann, E. \& Koshino, M. The electronic properties of bilayer graphene. Rep. Prog. Phys. 76, 056503 (2013).

24. San-Jose, P., Gorbachev, R. V., Geim, A. K., Novoselov, K. S. \& Guinea, F. Stacking boundaries and transport in bilayer graphene. Nano Lett. 14, 2052-2057 (2014).

25. Waldmann, D. et al. Robust graphene membranes in a silicon carbide frame. ACS Nano 7, 4441-4448 (2013).

26. Simon, S. \& Halperin, B. Explanation for the resistivity law in quantum Hall systems. Phys. Rev. Lett. 73, 3278-3281 (1994).

27. Kou, A. et al. Electron-hole asymmetric integer and fractional quantum Hall effect in bilayer graphene. Science 345, 55-57 (2014).

28. Weitz, R. T., Allen, M. T., Feldman, B. E., Martin, J. \& Yacoby, A. Broken-symmetry states in doubly gated suspended bilayer graphene. Science 330, 812-816 (2010).

29. Maher, P. et al. Tunable fractional quantum Hall phases in bilayer graphene. Science 345, 61-64 (2014)

30. LeRoy, B. J. \& Yankowitz, M. Emergent complex states in bilayer graphene. Science 345, 31-32 (2014)

\section{Acknowledgements}

We thank O. Pankratov for helpful discussions and D. Weckbecker for computational assistance. The work was carried out in the framework of the SFB953, the PP1459 and the Cluster of Excellence EXC 315 'Engineering of Advanced Materials', supported by the DFG. We acknowledge the support of the HLD at HZDR, a member of the European Magnetic Field Laboratory (EMFL).

\section{Author contributions}

F.K. and H.B.W. conceived the experiment. F.K. and C.O. carried out sample preparation, electrical measurement and data analysis, supported by C.H. in an early stage. F.K. and E.K. carried out high magnetic field measurements. B.B. and E.S. contributed structural information on dislocation networks by TEM. The quantum mechanical calculations were developed and performed by S.S. Network simulation was performed by F.K. and C.O. The manuscript was written by F.K., C.O., S.S. and H.B.W. All authors discussed the results and implications and commented on the manuscript at all stages.

\section{Additional information}

Supplementary information is available in the online version of the paper. Reprints and permissions information is available online at www.nature.com/reprints.

Correspondence and requests for materials should be addressed to H.B.W.

\section{Competing financial interests}

The authors declare no competing financial interests. 


\section{Methods}

Sample preparation. Hall bar devices were fabricated on commercial $6 \mathrm{H}-\mathrm{SiC}$ and $4 \mathrm{H}-\mathrm{SiC}$ wafers (semi-insulating, nominally on axis). To obtain an atomically flat substrate on the (0001) surface, samples were either hydrogen etched for $10 \mathrm{~min}$ at $1,520^{\circ} \mathrm{C}$ (for $6 \mathrm{H}-\mathrm{SiC}$ ) or annealed for $30 \mathrm{~min}$ at $\sim 1,700^{\circ} \mathrm{C}$ and $\sim 900 \mathrm{mbar}$ Ar pressure in a SiC container (for $4 \mathrm{H}-\mathrm{SiC}$ ). Graphene was grown for $15 \mathrm{~min}$ at $\sim 1,750^{\circ} \mathrm{C}, 900 \mathrm{mbar}$ Ar pressure, followed by a hydrogen intercalation at $\sim 900^{\circ} \mathrm{C}$ for $\sim 60 \mathrm{~min}$. Hall bar devices were fabricated by two standard e-beam lithography steps, one using $\mathrm{O}_{2}$ plasma etching, defining the shape of the Hall bar, with the second for evaporation of $\mathrm{Ti} / \mathrm{Au}$ contacts.

Network simulation. The resistor network was simulated according to the PL model, accounting for boundary conditions and Kirchhoff rules. Technically similar, a network consisting of three-terminal interconnected discs was investigated. To bridge the gap between the model and the real structure in the sample, parameters for each disc can be set independently (a resistance $R$ for interconnects and charge mobility $\mu$ and charge density $n$ for a disc). Short cuts and defects were introduced by introducing local $n=0$ and $n=\infty$ interconnect parameters into the network. Thus, the symmetry of the network is broken, reducing edge effects that arise from the number of terminals (even or odd) which are connected to the leads.
Electrical transport measurements. Magnetoresistance measurements up to $8 \mathrm{~T}$ were carried out in a ${ }^{4} \mathrm{He}$ bath cryostat with an embedded $1 \mathrm{~K}$ pot (sample in vacuum) using a standard, low-frequency lock-in technique. Electrical transport measurements in a high magnetic field were performed at the Dresden High Magnetic Field Laboratory in a $62 \mathrm{~T}$ and a $70 \mathrm{~T}$ pulsed field magnet, both with a pulse duration of about $150 \mathrm{~ms}$. The sample temperature was controlled using a ${ }^{4} \mathrm{He}$ flow cryostat (sample in He atmosphere) with an additional local heater for temperatures at room temperature and above. In one experiment (Supplementary Fig. 1a,b) an a.c. current of $1 \mu \mathrm{A}$ and a frequency of $1-2 \mathrm{kHz}$ was applied to the sample and all voltages were recorded using a $1 \mathrm{MS} \mathrm{s}^{-1} 16$-bit Yokogawa DL750 oscilloscope. The sample resistances were calculated by applying a digital lock-in procedure to the recorded data. In a second experiment (Fig. 1b) a d.c. current of $\sim 10 \mu \mathrm{A}$ was applied to the sample and the resulting voltages over the sample were recorded with a DL750 oscilloscope.

Quantum theoretical calculations. The effective field is constructed as described in the Supplementary Information and the quantum calculations are then performed using a basis of single-layer states-we find that up to 20,000 such states are required for full convergence of the problem to be achieved. This results in an $80,000 \times 80,000$ matrix, which evidently requires parallel computation. We diagonalize this matrix via the usual ScaLAPACK approach, using 64 cores. The effective field must be transformed into the single-layer graphene basis; this is achieved via a fast Fourier transform. 